\title{
La decadencia de las lenguas autóctonas de Guinea Ecuatorial: una manifestación de la pérdida de identidad cultural
}

\section{The decline of the indigenous languages of Equatorial Guinea: a manifestation of the loss of cultural identity}

\author{
Pedro Bayeme Bituga-Nchama \\ Universidad Nacional de Guinea Ecuatorial, Bata, Guinea Ecuatorial \\ pedrobayem@gmail.com \\ https://orcid.org/0000-0003-2310-5879
}

Cruz Otu Nvé-Ndumu

Universidad Nacional de Guinea Ecuatorial, Bata, Guinea Ecuatorial

otunvecruz@gmail.com

https://orcid.org/0000-0001-9839-8595

(Recibido: 06/06/2021; Aceptado: 15/06/2021; Versión final recibida: 15/08/2021)

Cita del artículo: Bituga-Nchama, P. y Nvé-Ndumu, C. (2021). La decadencia de las lenguas autóctonas de Guinea Ecuatorial: una manifestación de la pérdida de identidad cultural. Revista Cátedra, 4(3), 35-56.

\section{Resumen}

El presente estudio se centra en el análisis de la situación sociolingüística de Guinea Ecuatorial. En este sentido, se parte del hecho de que la falta de aprendizaje de las lenguas autóctonas del país ha derivado en una pérdida de identidad cultural, la cual se ha visto agravada por otros factores como la globalización. La poca importancia que se presta a las lenguas autóctonas, hace que todas ellas estén sufriendo cambios que provocarían su decadencia y posterior desaparición. Se debe invertir en políticas lingüísticas que puedan contrarrestar este efecto porque cuando desaparece una lengua lo hace también una parte de la misma cultura. Es importante remarcar que esta investigación no se ancla en el relativismo lingüístico por cuanto reconoce que potenciar las lenguas autóctonas no es una manera de vetar las lenguas oficiales del país.

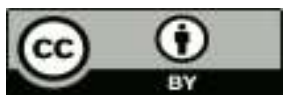


En la actualidad existe una pérdida de identidad cultural motivada porque no se enseña a los niños las lenguas autóctonas, pretenden que sean políglotas o bilingües sin una base lingüística propia: nos referimos a sus lenguas autóctonas que les identifican como parte de un grupo o comunidad. A lo largo de este trabajo, se pone de manifiesto que no puede haber políticas culturales sin políticas lingüísticas, porque las personas deberían conocer las lenguas autóctonas de su cultura. De hecho, los principales resultados de esta investigación evidencian la necesidad proteger las lenguas autóctonas, es incoherente potenciar el español en detrimento de las lenguas autóctonas que representan la identidad de cada grupo étnico.

\section{Palabras clave}

Autóctono, cultura, decadencia, identidad, lengua.

\section{Abstract}

This study focuses on the analysis of the sociolinguistic situation in Equatorial Guinea. In this sense, it starts from the fact that the lack of learning of the country's native languages has led to a loss of cultural identity, which has been aggravated by other factors such as globalization. The little importance given to indigenous languages means that all of them are undergoing changes that would cause their decline and subsequent disappearance. Invest should be made in language policies that can counteract this effect because when a language disappears, so does a part of the same culture. It is important to note that this research is not anchored in linguistic relativism because it recognizes that promoting indigenous languages is not a way to veto the official languages of the country.

At present there is a loss of cultural identity motivated by the fact that children are not taught indigenous languages, they pretend that they are polyglot or bilingual without their own linguistic base: we refer to their indigenous languages that identify them as part of a group or community. Throughout this work, it becomes clear that there cannot be cultural policies without language policies, because people should know the indigenous languages of their culture. In fact, the main results of this research show the need to protect autochthonous languages, it is incoherent to promote Spanish to the detriment of autochthonous languages that represent the identity of each ethnic group.

\section{Keywords}

Native, culture, decadence, identity, language.

\section{Introducción}

La presente investigación constituye un análisis sobre las lenguas autóctonas de Guinea Ecuatorial, porque se observa una pérdida sin precedentes de estas lenguas autóctonas o vernáculas, lo cual abre una brecha que conlleva a una crisis antropológica en nuestro contexto cultural. El propósito que nos conduce a estudiar esta realidad estriba en la importancia que es enseñar y utilizar las lenguas autóctonas para mantener la identidad cultural de los pueblos de Guinea Ecuatorial.

Por tanto, es evidente que la lengua confiere identidad cultural a sus hablantes. Antes de enunciar la tesis que se defiende en este trabajo, conviene justificar mejor por qué sostenemos que la lengua forma parte de la identidad de una persona o por qué la pérdida de la lengua implica pérdida de identidad cultural. En primer lugar, se ha de partir del hecho de que la lengua es uno de los elementos de una cultura, por lo tanto confiere una identidad a quien la habla. En este sentido, perder una lengua es perder la identidad cultural, porque 
la lengua es un sistema de comunicación donde se encuentra el pensamiento, las experiencias o incluso la historia del ser humano. Es importante que sea utilizada y se enseñe, independientemente de que la lengua tenga una grafía o no.

Por consiguiente, la tesis de la que se parte en esta investigación está en que, si se pierden las lenguas autóctonas, el hombre se convierte en un ser desconocido de su propio ser. Dicho de otra forma, la lengua esta inexorablemente vinculada a la cultura porque constituye un elemento de pertenencia para quien la usa. El respaldo teórico de esta afirmación la podemos encontrar en el hecho de que "la cultura y la lengua son aspectos indivisibles de la existencia total del ser humano" (Pozzo \& Soloviev, 2011, pp. 178-179). Desde luego, cabría deducir que, si se enseñan o potencializan el aprendizaje de las lenguas autóctonas, los niños conocerían mejor la realidad antropológica de su propia cultura, la cual se distribuye a través de las diferentes lenguas autóctonas. Así pues, la lengua constituye la realidad antropológica de una cultura y es un catalizador de la cohesión social.

A tenor de la tesis enunciada, hemos propuesto dar respuesta a los siguientes interrogantes: ¿Contribuyen las lenguas autóctonas en la difusión de la cultura? ¿Cómo afecta el desconocimiento de una lengua autóctona al ser humano? ¿Se puede superar la crisis de identidad cultural a través de la enseñanza de las lenguas autóctonas? ¿Existe una política lingüística en Guinea Ecuatorial?

En esta investigación se presenta como problema fundamental la pérdida de las lenguas autóctonas por la falta de aprendizaje de las mismas, causada principalmente por el impacto de la globalización y por las muchas lenguas que se reconocen como oficiales en Guinea Ecuatorial. Esta constante conlleva a una crisis de la identidad cultural, donde se observa que actualmente las nuevas generaciones de la sociedad ecuatoguineana, no hablan sus lenguas vernáculas como el fang, ndowe, bisio, etc. Este hecho pone en riesgo uno de los aspectos culturales de los grupos étnicos que componen ese país, nos estamos refiriendo a la identidad cultural.

En base en ello, nos motiva saber si la pérdida de las lenguas autóctonas implica perder la identidad cultural. Conviene enfocarse en el impacto de la globalización sobre las lenguas autóctonas de las culturas del mundo. Esta es la objeción de la que partimos en este trabajo, porque consideramos que la globalización no es un hecho aislado cuando se considera que se pierde la identidad cultural de un grupo étnico como es el caso de la lengua. No es un relativismo sociolingüístico, sino más bien la necesidad de que cada grupo humano conserve su cultura, y la lengua, que otorga identidad cultural, es parte de ella.

De entrada, queremos aclarar alguna cuestión relacionada con la lengua y la identidad cultural de la gente. Téngase en cuenta que más allá de la cultura que tenga cada uno, usamos las lenguas como un signos lingüísticos para comunicarnos, tanto con las personas de nuestra propia cultura (etnia, nacionalidad, etc.), como de otras culturas. Así, un hablante de fang de Guinea Ecuatorial, usará su lengua para comunicarse con las personas que también hablan esta lengua; pero esa misma persona podrá usar el francés, el español o el inglés, para comunicarse con otras personas que tienen otras lenguas, culturas u orígenes. Es decir, las lenguas, al facilitar la comunicación, favorecen el intercambio en la sociedad, ya sea esta familiar, local, regional, nacional, internacional. Hablar otras lenguas no significa necesariamente renunciar a la cultura de origen, usamos una lengua u otra para poder atender las necesidades que tenemos en cada momento.

"La lengua está íntimamente ligada al pensamiento, a la expresión de ideas, a la creatividad y a la cognición en general mediante una relación coarticulada y coevolutivamente desarrollada" (Piedra, 2010, p. 17). Y para aprender ese pensamiento, es necesario que cada 
cultura conserve su lengua. Lo que tampoco resulta una praxis normal es el hecho de que la lengua sea un instrumento de comunicación, se abandonen algunas lenguas para optar por otras que aparentemente son atractivas. Eso es, en otras palabras, aniquilar la cultura porque sin la lengua como signo lingüístico, sería difícil llevar a cabo, algo que para los seres humanos resulta vital, la trasmisión de la cultura de los pueblos, esa que les caracteriza y de alguna manera, forma parte de su esencia vital. En este sentido, es importante traer a colación el pensamiento de un destacado investigador que considera que:

[...] La lengua no es más que una parte del lenguaje, aunque esencial. Es a la vez un producto social de la facultad del lenguaje y un conjunto de convenciones necesarias adoptadas por el cuerpo social para permitir el ejercicio de esa facultad en los individuos (De Saussure, 1945, p.33).

En esa aseveración, De Saussure pone de manifiesto que hay una relación muy compacta entre la lengua y el lenguaje, por lo mismo que la hay entre lengua y cultura. El hablar da identidad y crea o mantiene al ser humano dentro de una estructura. Por tanto, el lenguaje, es lo humano, aquello que nos permite utilizar nuestro raciocinio y poderlo articular. En este sentido, el lenguaje es una ventaja natural del Homo Sapiens.

Por ello, cuando insistimos en que cuando una persona pierde su lengua se convierte en un ser desconocido de su propio ser, es para señalar que sin la lengua que da identidad a un ser humano, este se encuentra in albis, por desconocer su realidad antropológica o contextual del medio en que se desenvuelve, privándose de este modo, del pensamiento de esta cultura.

\section{Estado de la cuestión}

Abordar la cuestión de las lenguas autóctonas de la sociedad ecuatoguineana es una tarea que presenta grandes desafíos para cualquier investigador. El primer problema y el más fundamental, tiene que ver con la escasez de estudios sociolingüistas o etnolingüístas. Por ello, esta investigación tuvo que enfrentarse a esta gran dificultad. La temática vinculada al estudio de las lenguas siempre reviste de una gran importancia, por el hecho de analizar una dimensión importante del ser humano como es la lengua. La decadencia de las lenguas autóctonas de Guinea Ecuatorial es un fenómeno en el que intervienen muchos factores. Existen muchos argumentos por los cuales se puede considerar que la pérdida de una lengua implica una crisis antropológica sustancial.

Uno de estos argumentos se centra en señalar que:

[...] la actitud ante la lengua y su uso se convierte en especialmente atractiva cuando se aprecia en su justa magnitud el hecho de que las lenguas no son solo portadoras de unas formas y unos atributos lingüísticos determinados, sino que también son capaces de transmitir significados o connotaciones sociales, además de valores sentimentales. Las normas y marcas culturales de un grupo se transmiten o enfatizan por medio de la lengua (Fernández, 1990, p. 178).

Como se puede apreciar, nuestro lenguaje está en este sentido vinculado a la manera en la que somos. No es solo hablar bien, sino hablar para ser entendido y que entiendas aquello de lo que hablas.

El aprendizaje al que se somete el individuo desde niño, no es más que la capacitación en temas que tienen que ver con su cultura. No obstante, los aspectos que marcan sobre todo el lenguaje humano. En un mundo cada vez más cambiante, por la globalización como principal factor, hoy como nunca, es más importante abrir de nuevo o evocar un debate que 
a veces se suele pretender evadir a nivel internacional y es sobre las lenguas. Ante todo, hay que entender que todas las lenguas son igualmente importantes, por lo que ninguna es más importante que la otra independientemente de su naturaleza. El chino no es más importante que el francés o el inglés, como tampoco el portugués es más importante que la lengua bubi que se habla en Guinea Ecuatorial. No podemos caer en el etnocentrismo lingüístico.

Es importante precisar que aun cuando la lengua no sea lo único que otorga la identidad cultural a una persona, es un importante "soporte de la identidad cultural" (Fyle 1983, pp. 6-7).Sobre el debate de las lenguas, tenemos que alejarnos de cualquier pretensión etnocentrista, pues esto podría ofuscar o engañar al investigador, debido principalmente a que "[...] el etnocentrismo ordena la realidad social de forma jerárquica y establece criterios de superioridad e inferioridad respecto a los estilos de vida propios y de los demás" (Giner, 1998, p. 277). La mejor manera de proceder podría ser el reconocimiento de que cualquier pueblo tiene su propia lengua y que la imposición lingüística puede incluso acabar con la identidad de los habitantes de una determinada región, pues al perder su lengua, pierden su cultura y por tanto la identidad. Parece un eslabón que es mejor salvaguardar pues contiene la esencia del ser humano.

No estamos queriendo decir con ello que sea malo aprender otras lenguas, lo que nos llama la atención está en que cuando se aprende otra lengua, al mismo tiempo se aprende otra cultura, y se asimila como tal, de allí que el fenómeno de la globalización sea tan pujante, ya que arrasa las lenguas locales, imponiendo otra que termina absorbiendo la lengua autóctona del lugar. Dicho de otra manera, la globalización ha afectado de diferentes maneras la vida de las personas. "En el caso de la lingüística, este fenómeno ha ocasionado la hibridación, la evolución y en un caso extremo, la desaparición de lenguas y sus valores culturales que han estado en contacto históricamente con otros". (Villalobos Graillet, 2015, p. 5).

Este hecho se da de manera abismal actualmente, lo cual es un peligro para pequeños países o pequeñas comunidades, que ven cómo sus lenguas terminan desapareciendo. En este sentido, la lengua se presenta como "el reflejo del espíritu de un pueblo y está, por tanto, sometida a los avatares que el pueblo padezca" (Alvar, 1982, p. 39). Cuando desaparece una lengua, también lo hace la cultura, porque la lengua forma parte de la cultura, es identidad, y sin esta identidad, valga la redundancia, antropológicamente el hombre se convierte en ser desconocido de su propio ser. Al respecto, algunos autores investigadores sostienen que:

Las lenguas, como se sabe, distinguen a los pueblos, son portadoras de su sistema de valores culturales, constituyen un elemento importante de la nacionalidad como concepto, y son factor unificador [...] Si la globalización continúa su marcha actual predominante [...] sería el del idioma universal tantas veces soñado por filósofos y pensadores (Odio Zamora, 2001, pp.136-41).

La lengua es lo que cada uno de nosotros es, por eso, hay que hablarla y enseñarla a nuestros hijos para que ellos a su vez la enseñen a los suyos. La diversidad lingüística es un aspecto muy importante que hay que tener en cuenta, por lo mismo que las culturas y las personas son diferentes, hay que crear un mecanismo que impida o sirva de óbice frente a la globalización que fagocita de manera indiscriminada, las lenguas de comunidades pequeñas, simplemente porque las políticas nacionales no han sabido diseñar los causes necesarios para impedirlo.

El mismo concepto de cultura, como producto de la creación del ser humano, está vinculado de manera inexorable al de la identidad cultural. No quisiéramos redundar en la definición 
de lo que es la cultura, porque, al menos desde la perspectiva sociológica, encontramos que:

Alude al modo de vida de los miembros de una sociedad dada-sus hábitos y costumbres, junto a los bienes materiales que producen. Sociedad se refiere a los sistemas de interacciones que ponen en contacto a los individuos que comparten una cultura común. Ninguna cultura puede existir sin sociedad. Pero, por la misma razón, no puede haber una sociedad carente de cultura (Guiddens, 1991, p. 65).

Por eso, cuando uno se siente vinculado con un lugar en el que haya nacido o crecido, se identifica con él, es decir, con las costumbres y pautas de comportamiento del lugar, lo cual hace que se identifique o sienta que pertenece a ese grupo, porque comparte con él, ciertos rasgos que pueden ser creencias o simplemente valores culturales. En consonancia, Olga Lucia Molano sostiene que:

La identidad cultural de un pueblo viene definida históricamente a través
de múltiples aspectos en los que se plasma su cultura, como la lengua,
instrumento de comunicación entre los miembros de una comunidad, las
relaciones sociales, ritos y ceremonias propias, o los comportamientos
colectivos, esto es, los sistemas de valores y creencias (...) Un rasgo propio
de estos elementos de identidad cultural es su carácter inmaterial y
anónimo, pues son producto de la colectividad (Molano, 2008, pp. 69-84).

En esta definición de Gonzales Varas, la identidad cultural se imprime desde la cultura. Es decir, para identificarte con un grupo étnico, primero debes ser o conocer esta cultura. Cuando el mencionado autor habla de la lengua como uno de los aspectos de la cultura, lo hace sabiendo que es el instrumento de comunicación. Sin embargo, hay que precisar que lo que se comunica es la cultura, decimos comunicar porque se traspasa de generación a generación. Si no existe una lengua que identifique a un grupo étnico o que los miembros de este grupo étnico no le den tanta importancia a su lengua, entonces estamos o podemos estar ante una crisis de identidad cultural como ocurre con los grupos étnicos de Guinea Ecuatorial, cada uno de ellos en la medida de lo posible está perdiendo su lengua autóctona.

El abandono del aprendizaje y la enseñanza de las lenguas autóctonas se debe a múltiples factores, aunque como hemos destacado anteriormente, se debería principalmente al desdén por las mismas lenguas, por considerarlas pobres. Debido probablemente al hecho de que cada etnia utiliza y hace de su lengua lo que quiere. Sin embargo, existe un peligro real y está en que se está perdiendo un gran legado cultural. En este sentido, conviene refortalecer la identidad cultural, que como se ha planteado, hace referencia al sentimiento de pertenencia a un lugar o grupo.

Es posible afirmar que un pueblo tiene una identidad cuando sus individuos comparten representaciones en torno a tradiciones, historias, raíces comunes, formas de vida, motivaciones, creencias, valores, costumbres, actitudes y rasgos. Junto a ello deben tener conciencia de ser un pueblo con características diferentes a las de otros pueblos (Pérez et al., 1999, pp. 251-279).

En este sentido, cuando fallan todas estas cosas entonces existe una crisis de identidad cultual sin precedentes. Las lenguas autóctonas imprimen la identidad de cada uno de sus individuos. Si no se enseñan estas lenguas esta identidad está en gran peligro por la mera razón de no poder coexistir con las lenguas oficiales, aunque tampoco podemos sostener 
que exista una purga lingüística en el país, sino más bien que se debe revalorizar el papel de estas lenguas en la identidad cultural. Existe muchísima literatura sobre la sociolingüística, pero a nivel de la literatura académica nacional, apenas existen investigaciones sobre nuestro objeto de estudio. Esta es una de las razones por las que se ha tenido que usar un marco teórico de lo que se ha dicho a nivel académico en general, sobre la relación entre la lengua y la cultura, y sobre todo, la manera en la que la pérdida de la lengua, como uno de los aspectos de la cultura, afecta la identidad cultural.

\section{Defensa de la tesis}

El panorama de las lenguas de Guinea Ecuatorial no es muy alentador. Cada una de las lenguas de este país representa a los distintos grupos étnicos que podemos encontrar en él. La lengua es parte de la identidad cultural de una sociedad. El problema de Guinea Ecuatorial no son las lenguas oficiales reconocidas en la constitución, como es el español, francés y el portugués etc. El problema está en la actitud de rechazo o desdén con las que se conciben las lenguas autóctonas, que han pasado de ser una seña de identidad cultural, a un símbolo de bajo nivel social o económico, incluso se toma como falta de cultura universal.

No es descabellado pensar que las lenguas autóctonas viven una discriminación precisamente, por parte de los mismos ecuatoguineanos. Además, todos quieren que sus hijos no hablen estas lenguas, pues las consideran primitivas u obsoletas. Esta actitud tan negativa hacia las lenguas autóctonas es la que preocupa. No es en vano que Sanguinetti, haya sostenido que "el lenguaje es la casa del ser y en su morada habita el hombre" (Sanguinetti, 2006, pp. 59-72).

Cuando hablamos de lenguas autóctonas aquí, nos referimos sobre todo a las que son netamente del país. Es decir, aquellas lenguas que hablaban los nativos de Guinea Ecuatorial antes de la llegada de los europeos, y que sobrevivieron a la colonización. Actualmente, por citar algunas lenguas autóctonas en Guinea Ecuatorial:

Se hablan siete lenguas autóctonas [el bubi, el fang, el benga, el kombe, el baseke, el balengue, el bujeba] de la familia bantú, un criollo portugués [el annobonés], un pidgin inglés [el pichío pichinglis] y el español, como lengua general de coiné.No es un territorio monolingüe, ni siquiera bilingüe, sino que, como hemos visto anteriormente, es un crisol de lenguas, donde el español no es la lengua materna de ninguno de sus hablantes (Quilis y Casado-Fresnillo, 1995, pp. 27-35).

Lo más llamativo es el aspecto de la desaparición de las lenguas autóctonas. Actualmente, en Guinea Ecuatorial hay más hablantes de las lenguas oficiales que de las autóctonas. Probablemente esta ecuación no sea tomada en cuenta, pero la verdad es que uno de cada tres ecuatoguineanos se expresa en las lenguas oficiales del país, sobre todo en español. Decimos se expresa, porque el hecho de que hablen estas lenguas, no significa que todos lo hagan bien, es decir, que dominen los códigos lingüísticos o gramaticales de estas lenguas oficiales.

Además, la educación también juega un papel muy importante en el solapamiento de las lenguas autóctonas. En el $90 \%$ de los centros educativos del país se enseña en español, lo cual hace que los jóvenes hablen más español y fuercen a sus tutores o progenitores a expresarse con ellos en estas lenguas. Es sumamente preocupante esta asimetría que existe a nivel del país. La agonía que sufren las lenguas autóctonas de Guinea Ecuatorial solo refleja la crisis de identidad cultural. Sobre esta agonía a la que están sometidas las lenguas 
autóctonas de este país, Álvarez-Feáns, en una entrevista realizada a Justo Bolekia Boleká, señala que:

Una de las manifestaciones más claras de la enculturación se da a través de la lengua y cada vez es mayor el porcentaje de gente que no habla las lenguas nativas. La presencia de la lengua del colonizador en los contextos más destacados de la sociedad, como la escuela, la iglesia, los medios de comunicación hace que la gente de allí la identifique como aquella que les procurará lo que necesitan, la lengua del desarrollo, del prestigio, de la riqueza, y eso convierte a las autóctonas en lenguas enclaustradas y limitadas al contexto familiar [...] Cuando un africano no tiene ninguna lengua que le identifique como tal, sólo habla francés, inglés, español, portugués o alemán, deja de ser africano, porque la lengua es el soporte de nuestra identidad (Álvarez-Feáns, 2008, p. 35).

Los argumentos explícitos de Boleká solo son verdades que, a la luz de esta investigación, compartimos de manera rotunda. No decimos que se eliminen las lenguas extranjeras, sino que se proteja el legado cultural lingüístico de nuestros pueblos. Además, la observación directa sobre el contexto lingüístico de Guinea Ecuatorial, impela a tener que ser sensatos y admitir que hay una crisis antropológica, la cual puede ser entendida y explicada desde la antropología lingüística o la antropología filológica. Las lenguas autóctonas de Guinea Ecuatorial están necesitadas de mucho oxígeno, en el sentido de que ninguna de las lenguas habladas por los nativos de este país, puede considerarse exenta.

La crisis de identidad cultural causada por la pérdida de las lenguas autóctonas, se debe en gran parte a las endebles políticas lingüísticas. Para evitar este tipo de situación primero debemos partir del conocimiento de que "la cultura es lengua y lengua es cultura; se trata de una construcción simbólica particular de la realidad social; especifica nuestra comprensión de mundo y, por lo mismo, categoriza y valoriza nuestro accionar" (Teillier et al., 2016, pp. 137-161). Claramente, no se puede establecer relación con dependencia entre lengua y cultura si no hay lengua. Por ello, se ha de potenciar el aprendizaje de las lenguas nacionales para que la cultura siga en alza. Las características generales que se puede extraer de las lenguas autóctonas de Guinea Ecuatorial son las siguientes:

a) Todas son lenguas derivadas del bantú, excepto el annobonés, porque es una lengua criolla de origen portugués. Por bantú nos referimos a la gran familia lingüística de las lenguas que se habla en África.

b) Cada una de estas lenguas otorga una identidad cultural a sus hablantes, por lo que con el mero hecho de hablar una determinada lengua, se sabe directamente a qué etnia pertenece uno.

c) Son lenguas ágrafas. Hasta ahora, no existe evidencias de que haya existido alguna vez una grafía en las lenguas autóctonas de Guinea Ecuatorial. Sin embargo, existe algún lingüista que incluso ha publicado un diccionario en una de las lenguas autóctonas de las etnias del país. Se trata del escritor Julián Bibang Oyé.

El problema que existe con el diccionario de la lengua fang que ha publicado el mencionado autor, está en que no nos parece que se deba forjar a una cultura tener una grafía e imponerla a los hablantes, como constatamos en este caso. Lo mejor es llegar a un consenso con varios filólogos, etnolingüística, antropólogos, etc., para atreverse a constituir una grafía, lo impertinente es hacerlo de manera unilateral. Según se ha visto, el lenguaje puede ser verbal o escrito. En el caso de los pueblos africanos la mayor parte de las lenguas son ágrafas, es decir, carecen de escritura. 
Grosso modo, consideramos que estas son algunas de las características que podemos remarcar sobre las lenguas autóctonas. Téngase en cuenta que nos movemos entre la antropología lingüística y la antropología filosófica, por eso no queremos declinarnos tanto en el aspecto únicamente de la lingüística, aunque sea un tanto insoslayable. De entre las características que se ha anunciado anteriormente, solo podemos decir que se aprecia una pluralidad lingüística en función de los distintos grupos étnicos del país. Sin embargo, pese a esta pluralidad lingüística, cabe también destacar un matiz que pensamos que es importante. Si la cuestión que abordamos la emplazamos geográficamente, podemos decir que las lenguas autóctonas de Guinea Ecuatorial, sufren más decadencia en las zonas urbanas que rurales. No obstante, esto no significa que las zonas rurales estén exentas de este problema, pues las lenguas oficiales, sobre todo el español, es hablado con notoriedad en las zonas rurales, lo cual pone en peligro las lenguas autóctonas. No existe actualmente una cohabitación entre estas lenguas, más bien vemos que la cohabitación está desapareciendo poco a poco, porque las lenguas autóctonas ya se están dejando de enseñar y hablar.

Las diferentes lenguas que vamos a desarrollar no representan íntegramente el panorama lingüístico del país. El motivo de centrar nuestra atención en ellas, se debe principalmente a que son las más habladas.

\subsection{La lengua fang}

Al ser la lengua que más hablantes tiene, representa de esta manera la más golpeada o afectada por la pérdida o decadencia de esa lengua. Uno de los principales momentos de la pérdida de la lengua fang, se encuentra en la aculturación que sufrieron sus miembros debido a la colonización española. Sin embargo, con el paso del tiempo, no se ha podido recuperar parte de lo perdido.

La preocupación por resaltar que la lengua confiere identidad cultural a quien la habla, no ha pasado siempre desapercibido en Guinea Ecuatorial. De hecho, el presidente Francisco Macías Nguema, primer presidente del país, al ver que estaba en peligro la cultura fang por la invasión de la cultura europea, impuso el fang como lengua oficial del país porque es la que más hablantes tenía.

El sentimiento nacionalista fue una de las principales motivaciones que habrían llevado al presidente a imponer el fang como lengua vehicular, bloqueando de esta manera el español, pues, pensaba que iba en contra los valores culturales de los pueblos de Guinea Ecuatorial en general, pero en particular de los fang. De hecho, prohibió que se hablara más el español, algo que no fue posible que se cumpliera como tal. La pérdida de la identidad cultural de los fang, entendiéndose por tal, el hecho de ser fang, está sujeta a varios factores, cada uno de los cuales tiene un impacto muy considerable en esta lengua. Si bien la lengua fang es la más hablada del país, cabe mencionar que poco a poco está siendo invadida íntegramente por el español, lo cual pone cada vez más en peligro los vestigios culturales de este grupo étnico.

\subsection{Lengua bubi}

Después del fang, la lengua bubi es la segunda lengua autóctona más hablada en Guinea Ecuatorial. La mayor parte de sus integrantes se localizan en la región insular del país. El bubi no es una lengua en peligro de extinción, pero es posible que esté en este camino, pues hoy día se constata que la mayoría de ellos hablan más el pidgin, que es un inglés diferente al que estamos habituados, el cual aprendieron durante la estadía de los colonos británicos en la isla de Santa Isabel, hoy Malabo, capital de Guinea Ecuatorial. Sobre el uso del pidgin inglés en la isla de Bioko, Lipski señala que: 
Es considerado idioma vulgar, de clase social baja, mal hablado [...] donde los individuos hacen uso diario en la comunicación oral, en especial informal, del pichinglis más que de bubi, kombe o Annoboneses, pero el gobierno se niega a reconocerlo en sus actas. Su uso se ubica en especial en la capital Malabo, y menos en Bata (Lipski, 2004, pp.116-119).

En Guinea, el pichi o pichinglis forma parte de la realidad lingüística, sobre todo de la isla de Bioko, y sirve de instrumento de comunicación con nigerianos y cameruneses, que comparten este pidgin de base inglesa. Aparte del pidgin, también hablan el español, donde puede ser incluso la lengua materna de muchos de ellos. Existen bubis que nunca se han expresado en esta lengua, pues al desconocerla no pueden utilizarla, entonces, se encuentra en desuso. En la actualidad, la gente en edad senil son los que siguen siendo los garantes de la lengua de esta cultura, pues la utilizan para todo, es su lengua, por tanto, su cultura, y no es que no se quiera enseñar, sino que existe al igual que lo hemos dejado claro al hablar de la lengua fang, un rechazo por el aprendizaje de las lenguas vernáculas por parte de los jóvenes, aunque conviene decir que tampoco existen grandes intentos de mejorar esta situación. Conscientes de la pérdida cultural que se vive al no tener una lengua autóctona que salvaguardar, el profesor Bolekia Boleká, ha hecho varios intentos de presentar fórmulas para que los jóvenes se interesen por el aprendizaje de la lengua bubi.

\subsection{Lengua annobonesa}

La lengua annobonesa es, ante todo, la fusión de un idioma criollo, producto de la colonización portuguesa. En este sentido, no podemos decir que originariamente los annoboneses tienen una unidad lingüística. Más bien han desarrollado una lengua vernácula conocida como ambo, que aglutina a todos los annoboneses, ya que es una seña de identidad cultural para ellos. Además, probablemente, la situación geográfica de Annobón puede estar influyendo mucho en el hecho de que la lengua annobonesa permanezca robusta. Si bien el español es la lengua oficial del país, y todos los ciudadanos que residen en el deben utilizar esta lengua como la de cohesión social, en la isla de Annobón se habla preferentemente $f a$ dambo, que es la lengua autóctona de esta zona.

\subsection{Lengua ndowe}

La lengua ndowe es la cuarta lengua autóctona más hablada del país. Sin embargo, tampoco se ha quedado al margen de la pérdida de la identidad cultural causada principalmente por el desconocimiento o la negación a la difusión y aprendizaje de la lengua. Esta lengua de la que hablamos es utilizada principalmente por los ndowe, sin embargo, conviene precisar que se trata de una lengua hablada por los playeros, es decir, los grupos étnicos que se encuentran en el litoral del país. Se trata de un grupo étnico compuesta por los combes, los baseques, etc. Por otra parte, conviene señalar que los ndowe entraron en contacto desde hace mucho con las lenguas occidentales, que terminaron introduciendo cambios culturales en este grupo étnico. Muchos ndowe como los combes, actualmente hablan más el español que su lengua autóctona. Entonces, ¿cómo pueden los ndowe pensar que se puede conservar su identidad, si la lengua que caracteriza esta lengua está en desuso?

De nuestra parte, pensamos que la lengua es cultura, por tanto, identidad, sin ello, no puede pensarse que existan grupos étnicos que digan ser de una etnia concreta cuando realmente no se expresan en su lengua autóctona. Las causas del rechazo o la falta de interés que sienten los ndowe para inculcarla a sus hijos son debidas a múltiples factores. Las políticas lingüísticas y culturales son las herramientas que el Estado debe poner a disposición de todos, para así evitar esa crisis de identidad cultural que afecta a todas las etnias del país, y 
que si no se detiene, se corre el riesgo de que la globalización termine llevándoselo todo a su paso, siendo desastrosos para las lenguas ágrafas como las nuestras.

\subsection{Lengua bisio}

De todos los grupos étnicos descritos con anterioridad, los bisio son uno de los menos numerosos, por lo que es, en primer lugar, un grupo étnico cuya cultura puede desaparecer. Si decimos cultura, es porque también lo hará la lengua. Probablemente, la vitalidad de una lengua se deba al número de hablantes con la que cuenta. Por vitalidad lingüística nos referimos al "uso real de la variedad por parte de una comunidad de hablantes nativos" (Mounin, 1979, p. 89). En el caso de los bisio, no se puede hacer alarde de ser una cultura cuya identidad cultural cuente con varios miembros que constituyan la misma. En este sentido, la lengua bisio puede desaparecer con el tiempo. De hecho, si hacemos un balance de todas las lenguas autóctonas de Guinea Ecuatorial, la lengua bisio tiene altas probabilidades de conocer su ocaso. Pero, ¿por qué ocurre esto?

Sin lugar a dudas, esto ocurre por la falta de difusión de la misma lengua. El hecho que haya pocos hablantes que utilizan una lengua, no implica una condición necesaria, aunque sí puede ser suficiente para su desaparición. Aun cuando una lengua tenga pocos hablantes, si los mismos procuran utilizarla y salvaguardarla, no habrá muchísimos problemas. Con este planteamiento hay algo que quizás puede traer malos entendimientos en esta investigación. El pensamiento de un grupo cultural reside en la lengua que utilizan. Por eso, la identidad lingüística y cultural van cogidos de la mano. Las lenguas de autóctonas de Guinea Ecuatorial poseen la realidad del pensamiento cultural de cada uno de esos grupos étnicos. Se debe salvaguardar ese legado, sin embargo, la identidad cultural de estas etnias se ve amenazada como venimos destacando.

La globalización afecta profundamente a las lenguas autóctonas porque todas ellas han perdido en gran medida sus valores culturales. Es muy importante redefinir lo que es la identidad cultural, y sobre todo lingüística. Es urgente que se adopten medidas culturales que eviten que las lenguas autóctonas se vean absorbidas por varios factores. Se ha de detener la pérdida de identidad cultural o lingüística, causada por la falta de enseñanza de las lenguas autóctonas, así como el aprendizaje de las mismas. La cultura une personas o comunidades, es como un paraguas en el cual nos cobijamos, por ello, es importante conocer la propia lengua.

Seguimos insistiendo en que la cuestión en este trabajo no es que se eliminen las lenguas nacionales, pues forman ya parte de nuestro patrimonio. Entendemos que las lenguas autóctonas del país son todas ágrafas carentes de escritura, por lo que pretender eliminar las lenguas que se consideran oficiales para que algunas de las lenguas autóctonas como el fang o el bubi se conviertan en oficiales, sería muy conflictivo, pues se tendría que investigar una grafía.

Las lenguas autóctonas imprimen la identidad de cada uno de sus miembros, aquellos que la utilizan. Si no se enseñan estas lenguas, la identidad cultural queda comprometida por no poder coexistir con las lenguas oficiales, aunque tampoco podemos sostener que exista una purga lingüística en el país, sino más bien que se debe revalorizar el papel de estas lenguas en la identidad cultural.

El impacto de las lenguas extranjeras sobre las lenguas autóctonas o locales en este sentido, es sumamente demoledor, pues el impacto no solo se queda en lo meramente lingüístico, sino que también sociocultural. La convivencia de las lenguas extranjeras con las autóctonas 
no es nada armoniosa, porque debido a que las primeras se utilizan más, muchas de las lenguas autóctonas están desapareciendo, porque cada vez más cuentan con muy pocos hablantes. Por ello, la UNESCO sostiene que:

Muchos pueblos indígenas, asociando su condición social desfavorecida con su cultura, han llegado a creer que no merece la pena salvaguardar sus lenguas. Abandonan su lengua y su cultura con la esperanza de vencer la discriminación, asegurarse un medio de vida y mejorar su movilidad social o integrarse en el mercado mundial (UNESCO, 2003, p. 2).

No significa que este proceso es irreversible, porque no lo es. Lo que falta es que se diseñen políticas lingüísticas que permitan o ayuden a inculcar a los jóvenes lo importante que es aprender las lenguas autóctonas, pues son las que permiten que esta gente comparta con otros de su entorno, unos rasgos propios que caracterizan a su comunidad o grupo étnico al que pertenecen.

Cuando se abandona una lengua es porque la gente del lugar probablemente valore más las lenguas oficiales, aunque estas mismas lenguas sean extranjeras, y por tanto, que contribuyan al ocaso de la identidad cultural. Aquellos niños que no conocen su lengua están en una encrucijada pues no sabrían nada de su propio pensamiento que forma su propia cosmovisión del mundo. Los niños que desconocen las lenguas de sus padres y abuelos, tienen serios problemas de inclusión e interacción con la comunidad que debería instruirles en todos los valores culturales de su pueblo. En este proceso, la lengua vernácula juega un papel sumamente importante porque confecciona la propia identidad del ser humano. Aprender nuestras lenguas es un beneficio para nuestra propia experiencia a lo largo de la vida, es ser y pertenecer a algo.

Uno de los problemas que a priori se observa con respecto a la pérdida de la identidad cultural, es sin lugar a dudas, la falta de políticas educativas nacionales orientadas a la potencialización del aprendizaje de las lenguas autóctonas, como parte del patrimonio cultural de los diferentes pueblos o grupos étnicos que componen lo que es Guinea Ecuatorial. Esta manera de proceder es la que desde nuestra perspectiva hace que se prefiera incluso el bilingüismo. Para esclarecer lo que es el bilingüismo, lo utilizamos en este trabajo entendiéndose que es "la disposición que tienen las personas de manejar al menos dos lenguas distintas; las cuales son capaces de manipular y mezclar para alcanzar sus necesidades de discurso, y expresar su identidad multicultural" (Araujo-Quiroz, 2013, pp. 189-204).

El bilingüismo es uno de los aspectos que actualmente se está dando en nuestra sociedad pues son cada vez más los niños que se educan en este tipo de educación. Los niños ecuatoguineanos deben hablar actualmente español, francés inglés y portugués, eso sería quizás una nueva manera de expresar su identidad multicultural, sin embargo, no así con la misma identidad cultural. A raíz de todo el cuerpo teórico expuesto, la objeción que planteamos va en contra de quienes señalan lo siguiente: es mejor que cada persona hable las lenguas que quiera porque así es más culto y tiene mejores posibilidades de trabajo.

Como contraargumento a esta objeción planteada, hay que reconocer que es bueno que uno sea políglota o bilingüe, es decir, que se maneje con solvencia en varias lenguas. No obstante, a criterio personal lo mejor sería partir primero con el aprendizaje de las lenguas autóctonas, ya que lo que tampoco puede ser es que se hable muchas lenguas en detrimento de las autóctonas, aquellas que se habla donde uno nació. 
La solución no es ser bilingüe sino más bien tener primero una formación de cuna que implica conocer la lengua autóctona, la de nuestros padres, para posteriormente aprender otra. Antes de adoptar cualquier política lingüística, se debe preservar y enseñar las lenguas autóctonas para que los niños puedan aprender su cultura a través del habla de la lengua materna. Todo eso, porque "el lenguaje puede ser el vehículo por el cual, en los diversos perfiles que presenta la vida, podemos colaborar para establecer un nivel comunicativo en lo social y en lo cultural" (Habermas, 1987, pp. 351-432). En líneas generales, nuestra postura atestigua que quien desconoce la lengua de sus padres o abuelos sufre una auténtica crisis de identidad ya que no se le puede ubicar en una determinada cultura o región.

El aprendizaje de las lenguas autóctonas es de suma importancia porque es así como el ser humano se conecta con su medio. Hoy en día han desaparecido o están cayendo las lenguas autóctonas de Guinea Ecuatorial debido a la influencia de lenguas extranjeras y de la globalización. De esta manera, se ha creado una verdadera crisis antropológica en la vida de los ecuatoguineanos. Cuando un fang o annobonés habla una lengua extranjera, que no estamos diciendo que sea algo diabólico, se puede observar que lo hace como una persona que intenta adaptarse a otra manera de ver o concebir el mundo, pues la lengua es vida. Enseñar a los niños una lengua, es hacer que aprendan una determinada cultura. Si queremos que los niños sepan de su cultura y no estén en esta crisis antropológica, deben necesariamente aprender su lengua, y por ende, su cultura. Al respecto, tenemos que tener en cuenta un hecho no aislado, y es que:

Cuando las personas se comunican en un idioma que, al menos para una de ellas, es extranjero, no es posible asegurar que los significados y valores compartidos con los que están imbuidos sean los mismos, cosa que se puede suponer cuando los hablantes se expresan en idiomas que comparten características similares. La tarea de adquirir un idioma tal y como es hablado por cierto grupo en particular significa aprender los significados, valores y prácticas de dicho grupo, expresados a través del idioma (Byram y Fleming, 1998, p. 12).

Con esto, es evidente que si nosotros queremos que los niños hablen las propias lenguas de sus progenitores debemos comenzar nosotros mismos valorando primero nuestra cultura. Hoy por hoy, parece una necesidad implementar el bilingüismo al que se ha hecho alusión en uno de los acápites de esta investigación.

\section{Metodología}

Al tratarse de una investigación que aborda una cuestión sociocultural como es la perdida de las lenguas autóctonas, se ha llevado a cabo una metodología cualitativa. Las razones que han llevado a la elección de esta metodología se deben a que "la investigación cualitativa tiene relevancia específica para el estudio de las relaciones sociales, debido al hecho de la pluralización de los mundos vitales" (Flick, 2004, p. 15). No obstante, aun cuando sea una investigación cualitativa, es susceptible de recibir una cuantificación, la cual nos ayuda, gracias a los instrumentos y técnicas de investigación, conocer las razones de esta pérdida de identidad cultural causada por la pérdida de las lenguas autóctonas. Además, téngase en cuenta que "la metodología cualitativa tiene un valor exponencial en los estudios sociales, en tanto que reúne una serie de características que hace que la investigación vaya más allá de la mera suma de técnicas para la recogida de datos" (Taylor y Bogdan, 1994, p. 20).

Si bien las lenguas no constituyen las únicas manifestaciones culturales de los pueblos, hay que saber que están vinculadas con el pensamiento humano. Como técnica de investigación 
se ha utilizado la encuesta y el instrumento ha sido un cuestionario mixto, que contiene preguntas cerradas y abiertas, el cual ha sido importante para la recogida de los datos que se analizan en esta investigación. Se ha elegido la encuesta porque es la mejor técnica que se ajusta a esta investigación y porque gracias a ella se ha obtenido información de la muestra seleccionada sobre el tema objeto de análisis. En base en problema de investigación y a las preguntas formuladas, se ha podido realizar unas encuestas a la población ecuatoguineana. Las preguntas formuladas se han hecho partiendo de la base de que todos los encuestados hablan las lenguas ancestrales del país. La muestra seleccionada es de 402 ciudadanos, los cuales se encuentran en las dos principales ciudades del país, nos referimos a las ciudades de Malabo y Bata.

\section{Análisis y discusión de los resultados}

Los resultados que presentamos a continuación, son fruto de la encuesta realizada sobre una población muy selecta, la cual está compuesta por los principales grupos étnicos de Guinea Ecuatorial, los cuales hemos mencionado a lo largo de nuestra argumentación. Pensamos que estos datos son importantes, pues es una parte de esta investigación que ha permitido comprobar o verificar los objetivos planteados en esta investigación. Para analizar los datos, se ha utilizado el paquete estadístico para las Ciencias Sociales (SPSS), para la medición de las preguntas cerradas.

\begin{tabular}{|c|c|c|c|c|c|}
\hline & & Frecuencia & Porcentaje & $\begin{array}{l}\text { Porcentaje } \\
\text { válido }\end{array}$ & $\begin{array}{l}\text { Porcentaje } \\
\text { acumulado }\end{array}$ \\
\hline \multirow[t]{4}{*}{ válido } & $\begin{array}{l}\text { Están } \\
\text { desapareciendo }\end{array}$ & 104 & 25,9 & 25,9 & 25,9 \\
\hline & $\begin{array}{l}\text { Se hablan menos en } \\
\text { comparación con las } \\
\text { lenguas oficiales }\end{array}$ & 173 & 43,0 & 43,0 & 68,9 \\
\hline & $\begin{array}{lr}\text { Son consideradas } \\
\text { como parte de cada } \\
\text { cultura, pero no } \\
\text { existen políticas } \\
\text { lingüísticas para } \\
\text { enaltecerlas }\end{array}$ & 125 & 31,1 & 31,1 & 100,0 \\
\hline & Total & 402 & 100,0 & 100,0 & \\
\hline
\end{tabular}

Cuadro 1 ¿Cuál es la situación actual que encuentras en las lenguas autóctonas?

Según esta pregunta, el 43\% de los encuestados, señala que la situación de las lenguas autóctonas es preocupante porque se hablan menos en comparación con lenguas oficiales como el español o el francés. Esto se ve claramente en los porcentajes de la pregunta número uno de este cuestionario donde se observa que cada vez más los diferentes grupos étnicos del país hablan muy poco sus lenguas autóctonas, eligiendo preferentemente expresarse en español, francés o inglés, por ello desaparecen las lenguas autóctonas y porque no hay políticas lingüísticas más consolidadas que eviten este hecho. 


\begin{tabular}{rlrrrr}
\hline \multirow{2}{*}{ Válido } & Frecuencia & Porcentaje & \multicolumn{1}{c}{$\begin{array}{c}\text { Porcentaje } \\
\text { válido }\end{array}$} & \multicolumn{1}{c}{$\begin{array}{c}\text { Porcentaje } \\
\text { acumulado }\end{array}$} \\
& Sí & 223 & 55,5 & 55,5 & 55,5 \\
& No & 178 & 44,3 & 44,3 & 99,8 \\
& & 1 &, 2 &, 2 & 100,0 \\
& Total & 402 & 100,0 & 100,0 & \\
\hline
\end{tabular}

Cuadro 2 ¿Las lenguas autóctonas deberían ser también oficiales?

Una de las cosas que se nota en muchos países africanos, es la exigencia de los nativos y de algunos etnolingüistas, en la posibilidad de que las lenguas autóctonas africanas sean oficiales. Es el caso que observamos en la respuesta dada por nuestros encuestados, donde el 55,5\% está de acuerdo con que las lenguas autóctonas sean oficiales, posiblemente sea por darle valor e importancia a las lenguas autóctonas tal como ocurre con las lenguas que son oficiales en muchos países africanos, pero que pueden considerarse también como lenguas extranjeras. Sin embargo, a la luz de esta investigación se ha de reconocer que, para que las lenguas autóctonas de los países sean oficiales como tal, debe disponer también de una grafía, algo que no está presente en muchas de ellas. Todas las lenguas autóctonas también deberían ser oficiales, aunque esto puede que entrañe otras dificultades sociales.

\begin{tabular}{llrrrr}
\hline \multirow{2}{*}{ Válido } & Frecuencia & Porcentaje & $\begin{array}{c}\text { Porcentaje } \\
\text { válido }\end{array}$ & $\begin{array}{c}\text { Porcentaje } \\
\text { acumulado }\end{array}$ \\
& La & 111 & 27,6 & 27,6 & 27,6 \\
Globalización & 214 & 53,2 & 53,2 & 80,8 \\
$\begin{array}{l}\text { La falta de } \\
\text { interés por la } \\
\text { cultura }\end{array}$ & 77 & 19,2 & 19,2 & 100,0 \\
La pobreza & & & & \\
antropológica & 402 & 100,0 & 100,0 & \\
Total & & & & \\
\hline
\end{tabular}

Cuadro 3. ¿Cuál de las siguientes razones contribuye en la pérdida de identidad cultural?

La pérdida de identidad cultural a la que se alude en este trabajo y que se evidencia desde los distintos grupos étnicos del país, hace que la gente afirme que el 53,2\% se debe a la falta de interés por la cultura. Los motivos de esta falta de interés por la cultura pueden deberse a varios hechos no aislados, como la globalización que impone de manera directa, lo que deben adoptar sobre todo aquellas culturas minoritarias y que suelen terminar absorbiendo la cultura de otros pueblos. 


\begin{tabular}{|c|c|c|c|c|c|}
\hline & & Frecuencia & Porcentaje & $\begin{array}{l}\text { Porcentaje } \\
\text { válido }\end{array}$ & $\begin{array}{l}\text { Porcentaje } \\
\text { acumulado }\end{array}$ \\
\hline \multirow[t]{5}{*}{ Válido } & Ndowe & 97 & 24,1 & 24,1 & 24,1 \\
\hline & Bubi & 113 & 28,1 & 28,1 & 52,2 \\
\hline & Fang & 83 & 20,6 & 20,6 & 72,9 \\
\hline & Annoboneses & 109 & 27,1 & 27,1 & 100,0 \\
\hline & Total & 402 & 100,0 & 100,0 & \\
\hline
\end{tabular}

Cuadro 4 ¿Cuál de las siguientes etnias habla poco su lengua autóctona?

Según el análisis que podemos desprender de esta tabla, se observa que de todos los grupos étnicos que aparecen en esta pregunta formulada a nuestro encuestado, la etnia annobonesa es la que habla poco su lengua autóctona, probablemente, eso se debe a que son un grupo muy reducido de hablantes o debido a su pasado histórico, el cual hemos descrito en este trabajo. A parte de esto, los datos que manejamos aquí nos reflejan que los bubis son la segunda etnia que habla poco su lengua, esto también porque en la isla de Bioko se habla más español o el pichinglis. También están los ndowe, que se expresan más en español que en sus lenguas autóctonas. El panorama que vemos con eso, es que los fang son el único grupo étnico que habla más su lengua, aunque eso no signifique que no se esté perdiendo.

\begin{tabular}{|c|c|c|c|c|c|}
\hline & & Frecuencia & Porcentaje & $\begin{array}{c}\text { Porcentaje } \\
\text { válido }\end{array}$ & $\begin{array}{l}\text { Porcentaje } \\
\text { acumulado }\end{array}$ \\
\hline \multirow[t]{14}{*}{ Válido } & Está ayudando a & 42 & 10,4 & 10,4 & 10,4 \\
\hline & fortalecer la & & & & \\
\hline & cultura & & & & \\
\hline & ecuatoguineana & & & & \\
\hline & Está absorbiendo & 291 & 72,4 & 72,4 & 82,8 \\
\hline & las lenguas & & & & \\
\hline & autóctonas $\quad \mathrm{y}$ & & & & \\
\hline & contribuye en su & & & & \\
\hline & desaparición & & & & \\
\hline & Está coexistiendo & 69 & 17,2 & 17,2 & 100,0 \\
\hline & con las lenguas & & & & \\
\hline & autóctonas sin & & & & \\
\hline & alterarlas & & & & \\
\hline & Total & 402 & 100,0 & 100,0 & \\
\hline
\end{tabular}

Cuadro 5 ¿Cuál crees que es la relación del español con las lenguas autóctonas?

Al ser el español la lengua más hablada por los habitantes de Guinea Ecuatorial, hicimos esta pregunta para saber cómo la ciudadanía ve la situación del español con respecto a las 
lenguas autóctonas. Para el 72,4\%, la lengua española está absorbiendo las lenguas autóctonas, lo cual hace que se acelere la desaparición de las mismas, pues digamos que el número de hablantes del español en la sociedad ecuatoguineana es cada vez mayor en comparación con los tiempos pasados, sin embargo, este español tiene sus peculiaridades, pues es un español hablado en África, desde la cultura africana y tiene sus propias manifestaciones. De esta manera surge lo que podemos denominar español de Guinea, que es la fusión de las lenguas autóctonas del país con el mismo idioma español.

\begin{tabular}{llrrrr}
\hline & & Frecuencia & Porcentaje & $\begin{array}{c}\text { Porcentaje } \\
\text { válido }\end{array}$ & $\begin{array}{r}\text { Porcentaje } \\
\text { acumulado }\end{array}$ \\
Válido & $\begin{array}{l}\text { A través de } \\
\text { políticas } \\
\text { lingüísticas }\end{array}$ & 69 & 17,2 & 17,2 & 17,2 \\
inclusivas & & & & \\
A través de un & 182 & 45,3 & 45,3 & 62,4 \\
sistema & & & & \\
educativo & 151 & 37,6 & 37,6 & 100,0 \\
A través de la & & & & \\
propia familia & 402 & 100,0 & 100,0 & \\
Total & & & & & \\
\hline
\end{tabular}

Cuadro 6. ¿Cómo se puede fomentar el aprendizaje de las lenguas autóctonas en Guinea Ecuatorial?

Es evidente que las lenguas autóctonas deben ser potencializadas, y para ello, el Sistema Educativo Nacional se debe reorientar en la salvaguarda de nuestras lenguas. Es un peligro que las lenguas autóctonas nuestras no estén reconocidas en la Ley General de Educación vigente, como lenguas de enseñanza, que más bien lo son el español, francés, inglés o portugués. Uno de los resultados que se obtiene con este tipo de políticas, es que las lenguas extranjeras que son oficiales en este país, terminan o conducen al equipamiento de las lenguas autóctonas, porque si no se hablan en la escuela, en la familia o en las relaciones interpersonales, aquellas caen en desuso y terminan desapareciendo, pues no pueden adaptarse con las otras lenguas, porque no hay mecanismos creados para este cometido.

Así como existen instituciones oficiales del Estado como la Academia Ecuatoguineana de la lengua Española (AEGLE), también debería existir con urgencia una institución que salvaguarde el legado cultural de las lenguas autóctonas, el cual se encuentra impregnado en las diferentes lenguas autóctonas. Por otra parte, téngase en cuenta que para lo de las lenguas autóctonas, debe haber una confluencia de muchos factores, pero si bien se ha señalado al sistema educativo como se recoge la tabla, el peso de la familia también debe ser notorio. Pues la educación de los padres es sumamente importante, si ellos no enseñan a sus propios hijos a hablar en fang o bubi, esos tampoco lo harán con sus hijos. Si se da un escenario así esa lengua desaparecerá sin lugar a duda. 


\begin{tabular}{lccclc}
\hline & & Frecuencia & Porcentaje & $\begin{array}{l}\text { Porcentaje } \\
\text { válido }\end{array}$ & $\begin{array}{l}\text { Porcentaje } \\
\text { acumulado }\end{array}$ \\
Válido & Sí & 205 & 51,0 & 51,0 & 51,0 \\
& No & 197 & 49,0 & 49,0 & 100,0 \\
& Total & 402 & 100,0 & 100,0 & \\
\hline
\end{tabular}

Cuadro 7 ¿El bilingüismo puede ayudar a la pérdida de identidad cultural?

De entre todas las preguntas planteadas, la respuesta obtenida aquí de parte de nuestros encuestados, donde el $51,0 \%$ ha señalado que el bilingüismo puede ayudar a la pérdida de identidad cultural, supone un desafío para nosotros. Esta respuesta puede considerarse válida, porque si entendemos que hablar muchas lenguas en detrimento de otras, sobre todo aquellas que no se utilizan ni se reconocen a nivel internacional o global, esto supone que muchas de ellas desaparecerían y, por tanto, habría una aguda crisis antropológica. Por ello, el dominar más de dos lenguas extranjeras, es bueno, siempre y cuando esto no suponga ignorar la lengua de uno mismo que le da esa seña de identidad y de pertenencia a un lugar concreto.

Teniendo en cuenta que el mundo está cada vez más interconectado y es una exigencia mundial, de manera indirecta, que la gente hablemos más de dos lenguas, pues aquellos que se expresen en una sola lengua, podrán ser considerados como los analfabetos de los tiempos modernos. No obstante, insistimos de manera incesante que en la medida en que uno se hace bilingüe, deber ser siempre desde la base del conocimiento de la lengua de su cultura.

Es sumamente loable que nuestros niños hablen o se expresen en muchas lenguas, pues esto significaría que son ciudadanos muy actualizados y pueden integrarse fácilmente en otras culturas diferentes a las suyas. Sin embargo, valga la redundancia, un bilingüismo que se haga a expensas de evitar el aprendizaje de las lenguas autóctonas que permiten identificar a uno, es un bilingüismo vacío o nulo porque atenta con la misma esencialidad de las personas.

\section{Conclusión}

La lengua tiene una relación inexorable con la cultura, por eso, es importante identificarse con ella. A lo largo de esta investigación se ha puesto de manifiesto que la lengua otorga una determinada identidad para quien la habla. Ahora bien, en vista de que las lenguas autóctonas se hablan muy poco, lo cual hace que estén desapareciendo paulatinamente, se está generando de esta manera una crisis de identidad antropológica.

Todas las lenguas tienen la misma importancia, pues son parte de la realidad de cada pueblo. En ella se encuentra todo el legado que se ha ido forjando desde los tiempos más remotos hasta la actualidad. La lengua de la gente se puede considerar viva siempre y cuando esté siendo utilizada por sus hablantes. Si no utilizan esta lengua que deberían ir transmitiendo de generación en generación desaparecerá. En este sentido, la situación lingüística del país es sumamente preocupante. De allí que exista una crisis antropológica causada fundamentalmente por la pérdida o el desinterés mostrado para el aprendizaje de las lenguas autóctonas. 
Se ha visto que cuando uno desconoce la lengua de sus progenitores o de su realidad social, se desconoce también a sí mismo. Con este trabajo de investigación no hemos dicho que los niños ecuatoguineanos no deban aprender las lenguas oficiales del país, más bien nuestro argumento es que deberían aprender primero su lengua autóctona que puede ser el fang, el bubi o el annobonés, por el mero hecho de que constituyen su propia base antropológica.

Con esta investigación, se ha revelado unos resultados que alertan sobre el peligro en que se encuentran estas lenguas. Prácticamente, sin excepción alguna, todas las lenguas autóctonas de Guinea Ecuatorial se hablan menos en el país, aunque hay que reconocer que existen etnias donde sí que se nota más el impacto de la pérdida de la lengua que en otras, la evidencia es que ninguna está a salvo de esta crisis de identidad.

No existe una cohabitación entre las lenguas autóctonas y las que son oficiales en el país. Ninguna lengua autóctona es oficial, por lo que están vetadas para ser utilizadas en ámbitos públicos, con el argumento de que así se evita que algún grupo étnico se vea discriminado por una supremacía lingüística ejercida por otro grupo étnico que se sienta con la autoridad para poder hacerlo. Mientras las lenguas extranjeras como el francés, español, inglés o portugués, sigan siendo las oficiales y se solapen las lenguas autóctonas, es evidente que se agravará con mucha más notoriedad la desaparición de estas lenguas. Por ello, si no se pueden oficializar las mismas como un efecto que evite que desaparezcan, al menos que se declare estas lenguas como parte de la realidad cultural.

Puede ser que oficializar las lenguas autóctonas no sea la solución al problema de crisis de identidad cultural, pero se debe explorar esta vía para ser si eso es factible o no, por ello, es imperativo que se diseñen políticas lingüísticas serias. Que se quiera admitir o no, la globalización está afectando muchos a las lenguas autóctonas del país. En este sentido, es el momento de impulsar la enseñanza de estas lenguas como nunca se ha hecho. Los niños desde temprano tienen el derecho de conocer las lenguas de su país, la que hablan sus padres, pero nos referimos a esa que aprendieron de sus padres.

Para los tiempos modernos, el bilingüismo viene muy bien, por cuanto permite que las personas tengamos más posibilidades de poder hablar una o más lenguas. Sin embargo, en este proceso del aprendizaje de múltiples lenguas, no se deben dejar atrás las lenguas autóctonas. Antes de aprender una lengua extranjera se debe conocer la lengua de base. No vaya a ser que tengamos políglotas que conozcan francés, inglés, chino o alemán, y desconozcan su lengua de base como el fang, el quechua o el catalán, por citar ejemplos.

Se necesitan políticas lingüísticas que fomenten el aprendizaje y el uso de las lenguas autóctonas de Guinea Ecuatorial. Los libros de texto que se utilizan en el sistema educativo del país están en castellano. Lo cual hace que el único lugar donde la niñez puede aprender la lengua autóctona de sus progenitores sea en la familia. Sin embargo, tampoco esto está siendo así, porque incluso en la familia se habla más español.

Una de las limitaciones de esta investigación está en la falta de estudios nacionales sobre el estado de estas lenguas autóctonas. A nivel académico no se le ha prestado tanta importancia a este problema. La mayor parte de las investigaciones como las citadas en esta investigación, se centran en el español hablado en Guinea Ecuatorial. 


\section{Bibliografía}

Araujo-Quiroz, C. B. (2013). "Bilingüismo: herramienta clave en el contexto universitario". Revista Historia de la Educación Latinoamericana. Vol. 15 №, 20, pp. 189 - 204.

Habermas, J. (1987). Teoría de la acción comunicativa. Madrid: Taurus.

Flick, U. (2004). Introducción a la investigación cualitativa. Ediciones Morata y Fundación Paideia Galiza.

Fyle, C. (1983). La lengua, soporte de la identidad cultural. El correo de la Unesco, Año XXXVI, Unesco, París, 6-7.

Quilis, A., \& Casado-Fresnillo, C. (1995). La lengua española en Guinea Ecuatorial. Madrid: UNED.

Álvarez-Feáns, A. Á. (2008). "Incluso estando callado puedes causar grandes problemas”. Revista Pueblos.

Piedra, L. (2010). Deixis personal y representaciones mentales: Propuesta de la existencia de los marcadores deícticos.cognitivos y su relación con la deixis personal (Tesis de maestría). Costa Rica: Universidad de Costa Rica.

De Saussure, F. (1945). Curso de lingüística general. Buenos Aires: Losada.

Alvar, M. (1982). La lengua como libertad y otros estudios. Madrid: Cultura Hispánica. Instituto de Cooperación Iberoamericana.

Giner, S. (1998). Diccionario de sociología. Madrid: Alianza.

Molano, O. (2008). Identidad cultural un concepto que evoluciona. Revista Ópera, (7), pp. 6984.

Odio Zamora, G. (2001). Globalización, lengua universal y traductores. ACIMED; 9 (2), pp.136-41.

Sanguinetti, G. C. (2006). Hermenéutica y tropología en carta sobre el humanismo de Martin Heidegger. Revista de filosofía, 62, pp. 59-72.

Pérez, M., Perryman, A., González, N., González, L., \& Abreu, M. (1999). Identidad nacional, organizaciones culturales y tiempo libre". Centro de estudios sobre la juventud. La Habana. jóvenes en los noventa, pp. 251-279.

Lipski, J. (2004). The Spanish Language of Equatorial Guinea. Arizona Journal of Hispanic Cultural Studies, Volume 8, pp.116-119.

Teillier, F., Llanquina, G., \& Salamanca, G. (2016). De qué hablamos cuando hablamos de etnolingüística: bases teórico-metodológicas para un trabajo con el mapunzugun. RLA. Revista de lingüística teórica y aplicada, 54 (2), pp.137-161.

Byram, M., \& Fleming, M. (1998). Perspectivas interculturales en el aprendizaje deidiomas: enfoque a través del teatro y la etnografía. Madrid: Cambridge University Press.

Taylor, S. J., \& Bogdan, R. (1994). Introducción a los métodos cualitativos de investigación. Barcelona: Paidós.

Pozzo, M. I., \& Soloviev, K. (2011). CULTURAS Y LENGUAS: LA IMPRONTA CULTURAL EN LA INTERPRETACIÓN LINGÜÍSTICA. Tiempo de Educar, 12(24), 171-205. 
Fernández, F. M. (1990). Metodología sociolingüística. Madrid: Gredos.

Villalobos Graillet, J. E. (2015). La globalización lingüística: el conflicto entre el inglés y el español. Glosas - Volumen 8, Número 7, 4-15.

Guiddens, A. (1991). Sociología. Madrid: Alianza Editorial.

Mounin, G. (1979). Diccionario de Lingüística . Barcelona: Labor.

UNESCO. (2003). Vitalidad y peligro de desaparición de las lenguas. Grupo especial de expertos sobre las lenguas en peligro convocado por la Unesco. Recuperado el 25 de Julio de 2021.2 Obtenido de http://www.unesco.org/new/fileadmin/MULTIMEDIA/HQ/CLT/pdf/LVE_Spanish _EDITED\%20FOR\%20PUBLICATION.pdf 


\section{Autores}

PEDRO BAYEME-BITUGA obtuvo en 2017 su título de Graduado en Humanidades, en el perfil de cooperación internacional y Desarrollo por la Facultad de Humanidades y Ciencias Religiosas de la Universidad Nacional de Guinea Ecuatorial. En 2020 obtuvo el título de Máster Universitario en estudios Humanísticos y Sociales de la Universitat Abat Oliba-CEU de Barcelona. Es doctorando en Humanidades para el Mundo Contemporáneo, en la línea de investigación de Historia y Sociedad, del programa de doctorado de la Escuela Internacional de Doctorado CEU (Universitat Abat Oliba-CEU de Barcelona). Actualmente es profesor titular de la Facultad de Humanidades y Ciencias Religiosas de la Universidad Nacional de Guinea Ecuatorial, donde imparte las asignaturas de Sexualidad, Género y Poder, y de Metodología de investigación científica. Sus principales temas de investigación incluyen las cuestiones feministas y culturales de su contexto. Además, es miembro cofundador del grupo pódium humanitas. Es autor de algunos artículos publicados en revistas de gran calibre científico como la Revista Cátedra, Asparkia, etc. Ha dirigido varios Trabajos Fin de Grado relacionados con sus líneas de investigación.

CRUZ OTU NVÉ-NDUMU, en el año 2000 obtuvo el título de Maestro Industrial en la rama de automoción; en 2017 obtuvo el título de Grado en Humanidades perfil Cooperación Internacional y Desarrollo Sostenible en la Facultad de Humanidades y Ciencias Religiosas de la Universidad Nacional de Guinea Ecuatorial. En 2019 cursó el curso de Expertos Universitario en Derechos Humanos por el Instituto Superior de Ciencia y Educación, España. En 2021 obtuvo el título de Máster Universitario en estudios Humanísticos y Sociales de la Universidad Abat Oliva CEU Barcelona.

Actualmente es funcionario del Ministerio de Industrias y Energía, donde ocupa el puesto de o Jefe negociador de fomento industrial en la sección de industria. En el campo de la docencia, ha pasado varios años impartiendo clases en el Instituto politécnico Modesto Gené Roing de Bata. Es además, profesor interino de antropología cultural en la Universidad Nacional de Guinea Ecuatorial. 